\title{
El reto de la descentralización educativa: reflexiones desde la miradade actores nacionales y subnacionales en el caso del departamento del Atlántico*
}

\section{The Challenge of Educational Decentralization: Reflections from the Perspective of National and Subnational Social Actors in the Case of the Department of Atlántico}

Juan David Parra**

Recibido: 8 de agosto de 2016

Aprobado: 29 de noviembre 2016

Publicado en línea: 10 de octubre de 2017

\section{Resumen}

Según teóricos de la escuela de la elección pública, la descentralización política y administrativa mejora la eficiencia en la prestación de servicios públicos territoriales. Este artículo parte de una visión crítica de este marco de referencia en el estudio del fenómeno del bajo desempeño escolar. La experiencia del departamento del Atlántico representa un caso atípico del cumplimiento de los supuestos de la primera escuela y, por tanto, abre la posibilidad de entablar una discusión ontológica y práctica sobre el estudio del proceso de descentralización educativa básica y media en Colombia. El uso de un paradigma realista como referente metodológico para analizar entrevistas individuales y grupales hechas en diferentes

\section{Abstract}

Public choice theorists argue that political and administrative decentralization increases the efficiency in the delivery of public services in local communities. This paper departs from a critical stand of this perspective in studying the phenomenon of poor school performance. The Department of Atlántico is a case that deviates from the assumptions of public choice scholars, and hence its analysis serves to open an ontological and practical discussion on how to study processes of primary and secondary educational decentralization in Colombia. The use of a realist paradigm as a methodological backup to analyse a set of individual and group interviews at different levels of government helps

doi:10.11144/Javeriana.papo22-2.rder

\footnotetext{
* Artículo de reflexión.

** Profesional en Gobierno de la Universidad Externado de Colombia, magíster en Economía por la Universidad de los Andes y doctorando en el International Institute of Social Studies, Erasmus University Rotterdam. Correo electrónico: parraheredia@iss.nl ORCID: http://orcid.org/0000-0003-1902-7660
} 
niveles de gobierno permite entrever varias de estas implicaciones. Temáticas identificadas como la capacidad limitada de autoridades locales para diseñar y administrar políticas educativas, la falta de claridad entre actores de distintos niveles del sistema nacional de educación sobre el uso de las pruebas de Estado y la dificultad que enfrentan rectores de instituciones educativas públicas para que se transfieran oportunamente recursos para la calidad educativa son indicativas de complejidades de la política educativa omitidas en los diagnósticos del sector publicados en la literatura dominante.

\section{Palabras clave}

elección pública; descentralización; educación; realismo crítico; Atlántico

\section{Cómo citar este artículo:}

Parra, J. P. (2017). El reto de la descentralización educativa: reflexiones desde la mirada de actores nacionales y subnacionales en el caso del departamento del Atlántico. Papel Político, 22(2), 339-367. https://doi.org/10.11144/ Javeriana.papo22-2.rder to unveil many of these implications. Themes identified in this study include the limited capacity of local authorities to design and administrate educational policies, the lack of clarity among the actors of different levels of the national education system about the use of state examinations, and the difficulty experienced by school directors of public educational institutions to promptly access educational quality resources. These findings are all indicative of policy complexities omitted in the diagnostics of the sector published in the mainstream literature.

\section{Keywords}

public choice; decentralization; education; critical realism; Atlántico 


\section{Introducción}

Pese a ser parte de un debate inconcluso, hay cierto consenso académico que señala una relación positiva entre formas de descentralización política y la prestación de servicios públicos en América Latina (Faguet, 2014). En Colombia, existe un acervo de estudios que corrobora la identificación de correlaciones importantes entre fenómenos como la competencia electoral o el aumento de los esfuerzos fiscales subnacionales y el incremento en índices de cobertura y matrícula escolar (Faguet y Sánchez, 2008, 2014; García, Espinosa y Parra, 2013; Sánchez y Pachón, 2013). Faguet y Sánchez (2014) argumentan la forma en que se valida con ello la pertinencia de la construcción de modelos teóricos de elección pública para explicar la mejora en indicadores de gasto e inversión como resultado del aumento de los incentivos subnacionales a la rendición de cuentas y la eficiencia administrativa. Su punto de partida es, por tanto, definir la descentralización como

la devolución por parte del Gobierno central de la autoridad sobre funciones específicas, con todos los atributos administrativos, políticos y económicos que estas implican (p. ej.el recaudo de impuestos, el gasto y la toma de decisiones) a gobiernos locales democráticos que son independientes del centro dentro de un dominio geográfico y funcional legalmente delimitado (las traducciones son mías). (Faguet y Sánchez, 2014, p. 229)

Una parte importante de los estudios citados se encuentra sustentada en visiones nacionales y tienen una clara orientación estadística sobre muestras representativas. Ello tiene posibles ventajas (p. ej. la validez externa de tendencias cuantitativas), pero también implica retos metodológicos en la identificación de explicaciones causales como ¿qué explica los resultados positivos o negativos de los modelos de descentralización en diferentes países o regiones? (Krauss, 2016). Frente a este segundo aspecto, Faguet (2014) reconoce que una porción importante de los esfuerzos académicos destinados al estudio de la descentralización y la gobernabilidad ${ }^{1}$ se ha centrado en análisis tipo insumo-producto - la búsqueda de correlaciones entre niveles de descentralización y diversas variables de intereses para hacedores de política- y muy poco en "las relaciones dinámicas subyacentes que deben estar operando para que se cumplan los supuestos de análisis (más) estáticos” (p. 10). Siguiendo una línea de argumentación similar, Grindle (2007) sostiene que las motivaciones de los actores políticos son complejas, lo cual hace necesario acudir a teorías de comportamientos más sustentadas en la sociología

${ }^{1}$ Gobernabilidad, según Faguet (2014), hace referencia a la acción o la manera de gobernar (n. 2). En este contexto, y de acuerdo con esta visión teórica, los indicadores referentes a la eficiencia del gasto público son un reflejo (o resultado) de cómo se gobierna en el ámbito local. 
y el análisis político para tener una mirada más integral de la evolución de procesos sociales (como la descentralización educativa). ${ }^{2}$

Este artículo contribuye a esta discusión y a la literatura sobre la descentralización educativa en general al develar interacciones complejas entre actores nacionales y subnacionales y la forma en que ellas impactan (o dan forma a) las dinámicas educativas locales. El punto de partida es diferente del de autores de la elección pública, cuya orientación epistemológica privilegia la predicción de resultados empíricos (p. ej. la variación en indicadores del sector educativo) para validar construcciones teóricas deductivas basadas en supuestos universales del comportamiento humano (p. ej. las personas son o tienden a ser racionales). La crítica, y la base de la propuesta empírica del artículo, se inspira en los postulados ontológicos del realismo crítico y su defensa a la naturaleza explicativa, y no predictiva, de las ciencias sociales (Parra, 2016, 2018). Para Archer (2009, 2013), Willmott (1999) y Patomäki (2009), todo análisis de causalidad (en ciencias sociales) debe contemplar el papel de la agencia humana como mediadora de las propiedades de las estructuras sociales (p. ej. los posibles incentivos de la política educativa) de los contextos históricos y temporales en que habitan individuos y grupos sociales. ${ }^{3}$ Una epistemología realista para el análisis de la descentralización educativa debe integrar, por tanto (al menos), dos elementos metodológicos: 1) reconocer la limitación de estudiar la causalidad como una simple expresión de condiciones necesarias (p. ej. la existencia de incentivos adecuados para un comportamiento eficiente) y 2) dar paso al estudio tanto de las interpretaciones subjetivas (p. ej. la lectura del contexto según diferentes actores sociales) como de las condiciones estructurales (p. ej.

\footnotetext{
${ }^{2}$ En su reciente revisión de la literatura sobre el estudio de la democracia subnacional en Colombia y América Latina, Pino (2017) identifica dos grandes corrientes que informan sobre el trabajo de investigadores colombianos al respecto: 1 ) el sistema de partidos y el clientelismo local y 2) la relación democracia- violencia política. Ontológicamente hablando, y sobre todo en caso de la primera corriente, prima aún una visión informada por la escuela de la elección pública que se critica en este artículo (p. ej. el estudio sobredimensionado de la relación entre competencia electoral y el buen gobierno en el territorio). Temáticamente, existe un sesgo en los estudios académicos producidos en Colombia que reproduce "una importación acrítica y descontextualizada de categorías conceptuales y supuestos teóricos explícitos e implícitos provenientes de la literatura sobre casos federales" ( $p$. 234). Por tanto, y en sintonía con el contenido de este artículo, el autor recomienda "no centrarse únicamente en el análisis de las unidades regionales, sino también tener en cuenta la importancia de unidades locales como los municipios en el desarrollo de la democracia subnacional, especialmente en los Estados unitarios" (p. 236). ${ }^{3}$ En términos realistas, esta visión ontológica corresponde al principio del dualismo analítico en el debate estructura-agencia en ciencias sociales. Para Willmott (1999), la referencia a la mediación humana (o agencial) de propiedades de estructura sociales indica que estas segundas funcionan a través de la acción humana, y no a pesar de ella. Este autor discute el ejemplo de un docente que opta, por ejemplo, por no cumplir a cabalidad tareas propias de su papel como actor de la política educativa (p. ej. no asistir a clase, dictar contenidos que no corresponden a currículos preestablecidos). Una conducta rebelde podría acarrear sanciones (estructurales) para el educador, que se materializarían, sin embargo, dependiendo de las relaciones humanas (o agenciales) que delinean la manera en que este interactúa con su entorno (p. ej. relaciones docente-rectores, docenteestudiantes). Esto no implica que las propiedades estructurales se reduzcan (o se equiparen) a la conducta humana o que carezcan por sí mismas de poderes causales ( $p$. ej. el poder de direccionar a los individuos hacia cierto tipo de comportamiento como el del buen docente). Sin embargo, la causa eficaz (o la activación de esos poderes causales) depende de las actividades de los seres humanos.
} 
las especificidades históricas de distintos contextos) detrás del surgimiento de resultados observables de gobernabilidad en el territorio.

La reflexión empírica de este trabajo surge del análisis de información secundaria (estadísticas oficiales) y primaria (entrevistas recogidas en diferentes niveles de gobierno) alusivas al caso del departamento del Atlántico. Como se discute más adelante, este representa una experiencia atípica del cumplimiento de los supuestos del modelo ideal (de la elección pública) de descentralización y, por tanto, abre una oportunidad para entablar un debate práctico y ontológico sobre el estudio de reformas educativas orientadas a mejorar la calidad de la educación en Colombia. A diferencia de autores que se centran en la identificación de regularidades empíricas (p. ej. correlaciones de variables), el enfoque realista supone dar énfasis al análisis de situaciones contingentes (no necesariamente predecibles, específicas a ciertos contextos) que surgen de interacciones complejas entre actores sociales reflexivos (o no necesariamente racionales ${ }^{4}$ ). Según advierte Patomäki (2009), una postura realista frente a la causalidad implica, por ende, un esfuerzo metodológico de interpretación por parte del investigador. Un objetivo central de este estudio consiste en interpretar visiones de distintos actores del sistema educativo a fin de profundizar en la comprensión de los retos de la política de educación básica y media en el país.

Las conclusiones del artículo surgen del estudio de tres subtemas identificados a partir del análisis de contenido de las entrevistas semiestructuradas y de grupos focales con funcionarios públicos, académicos, miembros de gremios de educadores, rectores y docentes. Por una parte, las percepciones expresadas permiten entrever una modernización restringida de las capacidades administrativas de las autoridades locales y la forma en que ello dificulta una focalización eficiente de recursos del sector educativo. Al estudiar el caso del uso de las pruebas de Estado (Saber 11), se identifica una fragmentación en los discursos y las expectativas sobre la calidad educativa entre niveles de gobierno, lo cual termina incidiendo, a juicio de diferentes actores entrevistados, en la creación de un currículo nacional que no responde a las necesidades educativas de cada territorio. Finalmente, según narran rectores y miembros de la Secretaría de Educación del Atlántico, debido a que la descentralización educativa hace parte de dinámicas más generales sobre el funcionamiento del Estado, la falta de rendición de cuentas en cuanto

\footnotetext{
${ }^{4}$ La noción de reflexividad citada se desprende de la ontología del realismo crítico y su orientación epistemológica a estudiar, y no asumir, el comportamiento humano. El análisis relacional sociológico, el cual critica el individualismo metodológico, implica que el comportamiento humano es resultado de múltiples causas que integran elementos psicológicos del aprendizaje social (tras la interacción con otras personas) y de posición estructural (la incidencia de la cultura o la situación material del individuo en el mundo) de actores y grupos sociales. Esta complejidad genera márgenes de no predictibilidad (o de la aparición de sucesos contingentes) y hace difícil simplificar la conducta humana a un modelo de racionalidad instrumental (Parra, 2016, 2018). Por tanto, este último tipo de racionalidad es tan solo parte del repertorio de las posibles motivaciones de las decisiones humanas (Henrich et al., 2001). El punto, nuevamente, es que todo comportamiento debe ser estudiado y no asumido de entrada.
} 
al uso de recursos para la calidad educativa pone en riesgo la coherencia y la pertinencia de proyectos educativos que se gesten desde el territorio.

Antes de proceder con la discusión resulta pertinente hacer una nota aclaratoria adicional sobre las limitaciones del estudio que se desprende de su postura realista. Dada la "imposibilidad de reducir todos los predicados explicativos (de fenómenos sociales) a términos individuales" (Archer, 2009, p. 58), es apenas previsible que un análisis que se enfoca solo en elementos de la agencia sea insuficiente para identificar una relación causa-efecto entre la descentralización política y la gobernabilidad educativa en el territorio. Este estudio se centra, por tanto, en parte de esa tarea (el análisis de elementos de la agencia) y, como tal, deja abierta la puerta para que se profundice en el estudio de las razones estructurales. ${ }^{5}$ De igual forma, es claro que centrarse solo en un caso limita la posibilidad de generalizar cualquier conclusión a un ámbito nacional. Sin embargo, el fundamento de esa crítica no puede ser simplemente estadístico. Si se aceptan los preceptos epistemológicos del realismo crítico, es claro que la causalidad econométrica es, en el mejor de los casos, una causalidad débil, lo cual hace que pierda peso el argumento de la llamada validez externa de los resultados. Dicho lo anterior, la riqueza de los estudios de caso comparados radica en su mirada a fondo de la interacción entre estructura y agencia en diferentes contextos. Este artículo es fiel a ese principio, lo cual implica que los resultados que surgen de un único caso son potencialmente más interesantes que el de estudios estadísticos a gran escala que carecen de un motor ontológico de explicación (Easton, 2010; Emmel, 2013).

\section{El estudio de la descentralización: un reto epistemológico}

Según un influyente estudio de la descentralización en Colombia y sus efectos sobre la provisión de servicios públicos en el territorio, el país se convierte en un caso emblemático del cumplimiento de supuestos teóricos de la literatura de la elección pública (Faguet y Sánchez, 2014). En palabras de sus autores, la lectura de hallazgos estadísticamente significativos se puede interpretar como el producto de dos factores:

(1) la mayor capacidad de respuesta de los gobiernos locales electos, que son capaces de identificar los bienes públicos con alto impacto marginal; y (2) la mayor demanda de rendición de cuentas que proviene del hecho de que la financiación venga de los bolsillos de los ciudadanos locales, lo cual, a su vez, tiende a generar una mayor eficiencia del gasto público. (Faguet y Sánchez, 2014, p. 246)

\footnotetext{
${ }^{5}$ Es relevante mencionar que este trabajo hace parte de una agenda de investigación más amplia enfocada en el estudio de la descentralización educativa en la Costa Caribe. Su lectura debe, por tanto, complementarse con la revisión de resultados de otros componentes del proyecto.
} 
Visto desde un plano ontológico, los teóricos de la elección pública asumen que los actores sociales son egoistas y, como tal, buscan siempre maximizar su utilidad individual (Martinez-Vazques, 2011). Esto incluiría a políticos y burócratas, quienes cuentan con incentivos para anteponer sus intereses al interés general. En tal medida, según argumentan Verger, Bonal y Zancajo (2016), se justifica la creación de arreglos institucionales que emulen las reglas del libre mercado y den paso a principios de administración pública orientados a la competencia y la rendición de cuentas. En la literatura de la descentralización, ello se traduce en la activación de mecanismos como la competencia política y la autonomía fiscal de gobiernos locales (Faguet, 2014). La lógica es relativamente simple: si los electores son racionales votarán por los mejores candidatos (llevando a que estos segundos compitan por esos votos), y dado que gran parte de la inversión pública sale directamente de sus bolsillos, los ciudadanos tendran mayores incentivos por monitear las decisiones de sus gobernantes.

Este marco teórico se reproduce con frecuencia en documentos insumo para orientar las políticas educativas en el país. Un ejemplo es el informe reciente publicado por la Fundación para la Educación Superior y el Desarrollo, en alianza con el Ministerio de Educación Nacional, en el cual se argumenta la importancia de girar recursos escolares directamente a entidades territoriales, "porque solo en el nivel local es donde se conoce en detalle los problemas del sector" (Villar, 2016, p. 157). Ello coincide con una de las recomendaciones de la Misión de Equidad de Movilidad Social contratada por el Departamento Nacional de Planeación respecto de la la necesidad de "avanzar más en esta descentralización, generando las condiciones para que sean los colegios los que tomen las decisiones relevantes sobre la educación que se imparte en el país" (Barrera, Maldonado y Rodríguez, 2014, p. 299). Puesto en estos términos, y en sintonía con la literatura de la elección pública aplicada a la educación, se justifica enfocar gran parte del debate en la definición de criterios de eficiencia y efectividad en la distribución de recursos públicos.

El estudio del caso de la descentralización educativa en el departamento del Atántico se convierte, sin embargo, en una oportunidad para discutir una excepción (en el mejor de los casos) a estos preceptos teóricos. Las estadísticas longitudinales computadas en la figura 1 sirven de preámbulo al análisis en cuanto representan una síntesis estadística del comportamiento de variables que, de acuerdo con la teoría de la elección pública, deberían tener una conexión empírica con indicadores de eficiencia escolar (vía los mecanismos discutidos en los párrafos anteriores). La variable de interés es el desempeño escolar, medida a través de resultados de pruebas estandarizadas (Saber 11). El hecho empírico por subrayadar es la forma en que, a pesar de que las variables fiscales, de inversión y de competencia en elecciones relevan tendencias y magnitudes muy similares entre el Altántico y el promedio de la nación, ello no ha 
mostrado una incidencia clara en disminuir brechas en resultados de las pruebas Saber 11 en la última década. ${ }^{6}$

\section{Figura 1. Indicadores escolares y descentralización fiscal y política.}
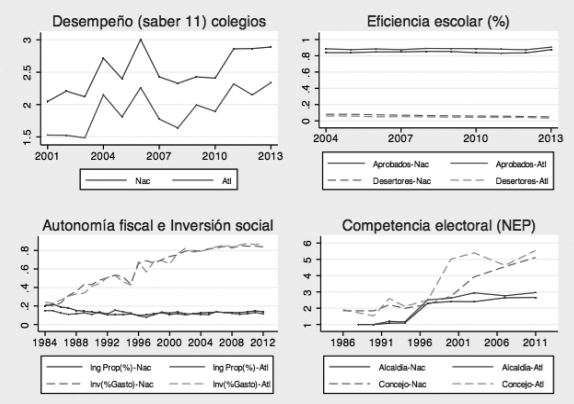

Nota: Los datos de eficiencia escolar corresponden a los reportes oficiales de aprobación y deserción escolar en municipios de Colombia. En la autonomía fiscal, se construyó un indicador de generación de ingresos propios (ingresos tributarios/ingresos totales), y para reflejar inversión social, se computó la proporción de formación bruta de capital sobre los gastos totales de cada municipio. Finalmente, como variable proxy a la competencia electoral, se utilizó el número efectivo de partidos políticos $(1 / \Sigma$ (proporción total de votos de cada partido) ^2) para elecciones a alcaldes y concejos municipales.

Fuente: Elaboración propia con datos del Instituto Colombiano para la Evaluación de la Educación, el Departamento Nacional de Planeación, el Banco de la República y la Registraduría Nacional del Estado Civil.

Desde un plano teórico, una forma de ver el problema consistiría en apelar a argumentos de estadística asintótica para justificar la preponderancia del análisis de la unidad promedio (p. ej. el municipio, el departamento) a fin de validar (o refutar) la robustez de los supuestos de la teoría de elección pública. Es decir, podría afirmarse que la existencia de un caso excepcional no es suficiente para invalidar una teoría, en cuanto en el agregado "la capacidad fiscal (que a su vez se correlaciona con dinámicas de competencia electoral) tiene un efecto significativo sobre el desempeño de los municipios en el sector de la educación” (Sánchez y Pachón, 2013, p. 27). En el campo de la econometría, son también frecuentes las discusiones en torno a las variables omitidas o los problemas de especificación de modelos estadísticos para estimar relaciones causaefecto entre variables de interés. En estos términos, las variables descriptivas cuentan

\footnotetext{
${ }^{6}$ Dentro del grupo de indicadores presentes en la figura 1, se incluye la capacidad agregada de los colegios municipales de evitar la deserción estudiantil. En todas las mediciones propuestas, las estadísticas del Atlántico siguen siendo similares a las del nivel nacional. Un estudio complementario como el de García, Espinosa, Jiménez y Parra (2013) muestra a su vez que no es claro que los colegios privados cuenten con una mayor capacidad de generar mejores resultados de desempeño escolar, como lo prevé la escuela de la elección pública (Verger, Bonal y Zancajo, 2016).
} 
con la restricción de presentar correlaciones altamente endógenas. Sin embargo, ello implicaría dar un estatus epistemológico a la estadística aplicada que, a juicio de autores como Krauss (2016), carece de sustento:

La idea teórica detrás de la causalidad probabilística presupone que las diferencias en probabilidad capturadas en un modelo estadístico deben tener una razón causal y que, bajo la condición de que sea posible controlar todos los demás factores (hecho que no es posible), la causalidad específica de interés debe ser la razón causal de la [relación observada] [...] al menos para la muestra recogida. Debemos acogernos a algunos supuestos para poder asumir que podemos derivar correlaciones o relaciones causales a partir de diferencias en probabilidad en un grupo de variables recogidas y que hacemos cuantificables [...]. En ese contexto, tiene poco sentido hablar de causas en lugar de la posibilidad de que dos fenómenos estén mutuamente correlacionados en el modelo estadístico y de que [solo] posiblemente se encuentren mutuamente correlaciones en el mundo real por fuera del modelo. (p. 102)

La crítica al análisis econométrico para el estudio causal de los efectos de la descentralización coincide con los elementos ontológicos del realismo crítico presentados en la sección introductoria del artículo. En particular, porque la omisión del análisis de la acción de individuos o grupos sociales frente a las propiedades (p. ej. los incentivos materiales y culturales) de las estructuras educativas que condicionan su comportamiento lleva a que los ejercicios estadísticos de gran escala dejen por fuera "las únicas causas eficientes de la vida social” (Archer, 2009, p. 272). Si bien es cierto que las estructuras educativas generan ciertos incentivos o rutas de comportamiento que trascienden la acción individual (p. ej. premiar a un buen docente y castigar al mal docente), estos poderes causales solo se materializan tras la acción y la interacción entre actores sociales como servidores públicos, rectores, docentes o estudiantes (Willmott, 1999; Patomäki, 2009; Parra, 2018). Siendo este el caso, el análisis de escenarios teóricos (como el que proponen los estudiosos de la elección pública) se concentran solo en una posible predicción de lo que podría suceder. En consecuencia, insiste Krauss (2016),

debemos abandonar el vocabulario que expresa causalidad, como aquel utilizado por académicos influyentes [...] sobre los "efectos causales" [...]. El dilema que surge es que el análisis estadístico tan solo puede proveer escasas probabilidades, mientras que la investigación que busca informar políticas públicas requiere mayores niveles de certidumbre y predicción, a pesar de que esto no es econométricamente posible. Dadas las limitaciones en hacer la democracia cuantificable y adaptable a investigaciones científicas, el estudio de las instituciones democráticas entre países es en general menos del tipo de la ciencia cuantitativa y más acorde con narrativas cualitativas. (p. 106) 
Visto desde esta perspectiva, el comportamiento atípico del caso del departamento del Atlántico cuenta con peso suficiente para apelar a la necesidad de adoptar rutas metodológicas alternativas para estudiar fenómenos sociales como el del bajo desempeño escolar. Las críticas esbozadas hasta el momento - sobre el determinismo ontológico que presuponen las teorías de elección pública ${ }^{7}$ y frente a la arbitrariedad estadística de la modelación econométrica- implican la necesidad de abordar el fenómeno de la descentralización como un proceso dinámico que se desarrolla en el tiempo y en contextos específicos, y que conlleva cadenas de decisiones complejas permeadas por interacciones entre oponentes y promotores de reformas educativas (Grindle, 2004; Patomäki, 2009). La teoría secuencial de Falleti (2005) cuenta con elementos de esta visión, en cuanto su distinción entre procesos de descentralización administrativos, fiscales y políticos representa un punto de partida para diseminar el análisis. Sin embargo, la vocación estructuralista (ambigua) de este lo lleva también a dejar a la agencia humana en segundo plano de importancia.

Estos preceptos conceptuales suponen adoptar una postura ontológica en torno a la forma en que las interacciones entre individuos y colectividades impactan el surgimiento de arreglos institucionales específicos. Trabajos como el de Archer (2009, 2013) y Willmott (1999), los cuales han tenido una incidencia importante en investigaciones contemporáneas sobre la evolución de los sistemas educativos en el mundo (Priestley y Miller, 2012; González, 2013; Edwards y Brehm, 2015), enmarcan esta reflexión en una posición realista del debate estructura-agencia. Por motivos de espacio no se presentará en detalle esta discusión, tarea que, sin embargo, hace parte de una etapa previa de esta investigación (Parra, 2016, 2018). No obstante, basta con citar un pasaje de la investigación de Archer (2013) sobre el origen de tres sistemas educativos europeos para identificar una ruta metodológica consecuente con una visión más compleja, dinámica e histórica de los fenómenos que hacen parte del interés académico de este artículo:

En lo que respecta al cambio, este no puede ser explicado hasta que no se dé cuenta del porqué cambian las metas educativas, quiénes hacen los cambios y cómo se imponen los cambios que buscan. Enfrentar estos problemas implica reconocer que su solución dependerá del análisis de formas complejas de interacción social. Más aún, la naturaleza de la educación raramente es [...] la realización práctica de una forma ideal de instrucción según es visualizada por un grupo en particular. Por el contrario, la mayoría de las veces las formas que adopta la educación son resultado político de pugnas por el poder [...] Por ende, para entender la naturaleza de la educación en un momento determinado, es

${ }_{7}^{7}$ Por determinismo se entiende la expectativa que indica que, dado que los individuos son maximizadores de utilidad, las fórmulas de mercado que apelan a esta racionalidad deben siempre dar un resultado eficiente. 
necesario reconocer, no solo quién ganó la pugna por el control, sino también quién la perdió y qué tanto perdió. (pp. 2-3)

La teoría social de Archer implicará, por tanto, que las formas de educación que se observan en un contexto geográfico y temporal específico son resultado, precisamente, del tipo y la forma de interacción entre grupos de interés. Esto es muy diferente del determinismo de visiones como las de la elección pública bajo las cuales se asume (al menos implícitamente) que las instituciones educativas operan en un vacío ontológico, y que son estas las que generan incentivos específicos a los actores sociales. En sintonía con el trabajo histórico y sociológico de Grindle (2004) sobre la reforma educativa en América Latina, lo que resulta relevante estudiar no es solo el tipo de instituciones que despliegan políticas educativas, sino la forma en que se negocian y se implementan ciertos arreglos institucionales.

La respuesta a interrogantes sobre fenómenos causales (p. ej. los efectos de la descentralización educativa sobre indicadores como el desempeño escolar) demandará, por tanto, un ejercicio que combine la descripción de percepciones expresadas por diferentes grupos de interés sobre los alcances de las reformas educativas, con la lectura de entornos materiales y culturales que sirven de contexto y que condicionan los intereses y las formas de interacción entre burócratas, asociaciones de maestros, académicos, entre otros (Archer, 2013). La tarea, citando nuevamente las posturas realistas enunciadas, no se reduce, sin embargo, a esta descripción, sino a la teorización sobre los procesos de mediación entre estructuras (los contextos y sus propiedades) y las agencias (individuos, grupos de poder) que dan surgimiento a resultados empíricos observables de diferentes procesos educativos. El objetivo de este artículo se centra en la primera etapa del análisis (la caracterización de los intereses expresados por los actores sociales), en cuanto la culminación del análisis implicaría la consulta de fuentes diferentes de las analizadas para su construcción.

\section{La hoja de ruta: el sueño de la descentralización educativa}

Antes de proceder con el análisis de las formas de descentralización que se han dado en Colombia, y en el caso específico del departamento del Atlántico, resulta conviente revisar la narrativa oficial de cómo se ha dado este proceso. Puesto en un plano teórico, y al menos desde una perspectiva de lo ideal, el objetivo de los procesos descentralizadores es replantear formas de gobierno, desde estructuras jerárquicas, burocráticas y altamente centralizadas, a sistemas anidados de autogobierno territoriales, caracterizados por mayores niveles de participación, cooperación y rendición de cuentas (Faguet, 2014). Pese a algunos matices, y el reconocimiento de algunos conflictos de facto, esta es la óptica genérica bajo la cual se ha descrito la reforma política y administrativa en el país. 
De acuerdo con algunos recuentos históricos (Barrera, 2005), es posible entender el estado actual de las políticas educativas en Colombia a partir de este marco referencia dominante. Como antecedente a las reformas de principios de la década de 1990, se habla de un proceso de transformación progresiva del Estado central en la segunda mitad de la década de 1970, con un incremento de la autonomía de las regiones subnacionales para recolectar impuestos y la apertura, unos años después, a la libre elección de alcaldes y gobernadores. Estos cambios, que se materializan con la firma de la nueva Constitución en 1991, implicarían un nuevo marco jurídico que asignaría una mayor responsabilidad a los gobiernos descentralizados para la provisión de bienes y servicios. Según autores como Hoyos y Ceballos (2004), Faguet y Sánchez (2008) y Falleti (2005), tal cambio de paradigma buscaba, dada la incapacidad de gestión del Gobierno central, dar respuesta a demandas territoriales por la redistribución de poder (primero) político y (luego) administrativo.

La Ley General de Educación (Ley 115/1994, de 8 de febrero) propone los principios básicos de la descentralización en la educación preescolar, básica y media en el país, que incluye estrategias administrativas para optimizar la relación estudiante-alumno, la contratación y la administración de cuerpos de docentes (regulada por el Decreto 1278/2002, de 19 de junio) y la financiación y asignación de recursos financieros. Este último tema se convertiría en antesala para la expedición de la Ley 715/2001, de 21 de diciembre y la fijación de nuevos criterios (i. e. basados en demanda educativa y no en indicadores de pobreza) en busca de una mayor eficiencia y equidad (entre municipios y regiones) en el gasto público (Iregui, Melo y Ramos, 2006; Ramírez y Téllez, 2007; Barrera, Maldonado y Rodríguez, 2014). Sin embargo, si bien este nuevo arreglo institucional es visto como un mecanismo exitoso para incrementar la cobertura educativa, para Villar (2016), "aún persisten falencias en el diseño del sistema, lo que no garantiza una asignación eficiente de los recursos para calidad" (p. 115).

La Ley 715/2001, de 21 de diciembre también contempla mecanismos para la profundización de la descentralización administrativa, respecto del delineamiento de deberes y responsabilidades de los diferentes actores del sistema educativo en el ámbito nacional y regional (Barrera et al., 2014). En tal medida, la nación estipula lineamientos y normas técnicas y curriculares, regula la prestación del servicio (que incluye el concurso para la carrera docente) y administra y asigna recursos (a través del Sistema General de Participaciones) que se giran a los territorios. Los departamentos y municipios certificados, por su parte, administran los recursos de dicho sistema destinados a mejorar la infraestructura escolar y la calidad educativa (la excepción son los recursos de gratuidad que son girados directamente a instituciones educativas y los recursos de calidad que son girados directamente a los alcaldes de todos los municipios), ${ }^{8}$ asignan plazas docentes y recopilan

${ }^{8}$ La suma de estos recursos, sin embargo, corresponden solo al $7 \%$ de la asignación total de los recursos del Sistema General de Participaciones (Villar, 2016). 
información (p. ej. evaluación de directivos y docentes) de las instituciones educativas regionales. Los rectores, por su parte, están encargados de ejecutar los proyectos educativos institucionales y evalúan el desempeño de los cuerpos docentes en los colegios.

En temas de diseño de políticas públicas, el sistema nacional de educación contempla que las secretarías departamentales de educación y los gobiernos distritales y municipales "pueden elaborar planes de desarrollo educativo o planes sectoriales de educación, dentro del Plan de Desarrollo aprobado por las asambleas departamentales y los concejos, respectivamente" (Ministerio de Educación Nacional [MinEducación], 2009, p. 32). Estos incluyen posibles iniciativas para atender necesidades específicas locales, y son financiados con una serie de recursos contemplados en la ley, que incluyen el residual de recursos del Sistema General de Participaciones para pago de gastos de funcionamiento y de salarios de personal (p. ej. salarios docentes) y los recursos propios generados por gobiernos regionales, inversión privada y recursos de cooperación, créditos, etc.

Volviendo al debate teórico, es también relevante hacer un breve recorrido por el contenido de algunas recomendaciones que se han hecho desde instancias académicas para fortalecer el proceso de descentralización educativa en el país. Se cita nuevamente el ejemplo de trabajos como el de Barrera et al. (2014) y Villar (2016), en la medida en que sus autores los presentan como producciones académicas orientadas a convertirse en insumos directos de políticas públicas. La síntesis, que puede hacerse citando un pasaje de cada uno de los documentos, da cuenta de la prevalencia de facto de los supuestos de marcos de referencia de la elección pública y la manera en que, como se ha podido ver a lo largo de esta sección, gran parte de la narrativa dominante del proceso de descentralización se hace a partir de una búsqueda unidireccional (desde el Estado central) por dar cumplimiento a principios rectores de desburocratización, uso eficiente de los recursos y, tras ello, la prestación de un mejor servicio en los colegios. En el primer caso,

en Colombia en este momento se debe hacer un esfuerzo grande para mejorar la calidad de la educación sin desatender la cobertura, como se mencionó anteriormente. [...] Las propuestas aprovechan la descripción de la reglamentación y las evaluaciones reseñadas tanto de programas desarrollados en el país como en otros países. Se hacen propuestas sobre seis temas: (i) fortalecimiento de la evaluación de la calidad educativa; (ii) fortalecimiento de la capacidad de gestión de los colegios; (iii) política de docentes; (iv) jornada escolar completa; (v) participación del sector privado en la provisión de educación; y (vi) fortalecimiento de programas exitosos. (Barrera et al., 2014, p. 295)

Por su parte, y en relación con uno de los subcomponentes referente al gasto público orientado a mejorar la calidad educativa, Villar (2016) recomienda 
i) otorgar mayor autonomía a las ETC [entidades territoriales certificadas en educación] y colegios en el uso de los recursos, ii) favorecer a aquellas ETC que produzcan ajustes/ mejoras en la estructura y calidad de la planta de personal, iii) favorecer a los colegios que hayan mostrado un buen desempeño, iv) incluir criterios de progresividad en la asignación de los recursos entre entidades territoriales, v) en los recursos orientados a los colegios, el objetivo de equidad se logra en el sentido de que la fórmula aísla el efecto que podría tener el hecho de que los colegios que atienden a niños de mejores condiciones socioeconómicas tienden a producir mejores resultados en términos de desempeño. (p. 237)

En este punto, vale la pena aclarar al lector que el objetivo de esta crítica no consiste en desestimar este tipo de miradas más administrativas de la descentralización, que sin duda son fundamentales para dar cumplimiento a metas fiscales y presupuestales que colindan con el interés general. No obstante, y de acuerdo con los postulados ontológicos discutidos en secciones anteriores, estos son diagnósticos muy generales que en muchos casos abren más interrogantes que los que resuelven (Archer, 2013) en cuanto reducen la complejidad de los problemas educativos a un recetario (algo) simplista y genérico (Verger, Bonal y Zancajo, 2016). En el resto del estudio, se introduce un análisis sobre fuentes primarias de información en busca de - y con lineamientos metodológicos realistas- empezar a dar sentido al mundo de complejidad que permiten tener una mejor comprensión de las dinámicas educativas nacionales y regionales.

\section{La descentralización de facto vista desde el caso del Atlántico}

Desde una óptica subnacional, la lectura detallada de documentos oficiales de política (Gobernación del Atlántico, 2014) y de informes producidos por centros académicos como el Observatorio de Educación de la Universidad del Norte (Chamorro, Borjas y Valencia, 2013; Said-Hung, 2014) relevan la fuerte alineación entre políticas educativas regionales y nacionales. A pesar de la existencia de matices en su contenido, es bastante evidente la coincidencia de objetivos priorizados (al menos en el papel) entre autoridades del departamento y de la nación. Sin embargo, siguiendo a analistas como Vivas (2009) y Henríquez (2013), ello parece ser más reflejo de la ausencia de iniciativas educativas regionales por cuenta del hecho de que "la autonomía efectiva de los municipios y departamentos afronta [...] serias dificultades en la generación de los ingresos necesarios para el impulso definitivo de la calidad educativa” (Vivas, 2009, p. 172).

Esta última hipótesis sirve de punto de entrada para el análisis de contenido sobre entrevistas individuales y grupales con actores educativos nacionales y subnacionales (tabla 1). ${ }^{9}$ La recolección de información en campo, que incluyó una exploración et-

${ }^{9}$ Todo este material fue transcrito y codificado utilizando el software cualitativo NVivo. El análisis partió del uso de las categorías de tipo de descentralización (administrativa, fiscal y política) de Falleti (2005), y se combinó 
nográfica en instituciones educativas de dos municipios del sur del departamento del Atlántico - seleccionados porque representan casos de persistencia en bajo desempeño escolar- se hizo entre agosto de 2014 y julio de 2015. Si bien el objetivo de este artículo no es presentar los resultados globales de la investigación, se mencionan estos detalles metodológicos para dar cuenta de la operación de un enfoque realista orientado no solo a observar, sino también a contrastar información y poner a prueba postulados teóricos en campo (Pawson, 1996; Easton, 2010; Emmel, 2013; Rees y Gatenby, 2014).

Tabla 1. Lista de actores entrevistados.

\begin{tabular}{|c|c|c|c|c|}
\hline Cargo & Tipo de institución & $\begin{array}{c}\text { Nivel de } \\
\text { gobierno }\end{array}$ & $\begin{array}{c}\text { Tipo } \\
\text { entrevista }\end{array}$ & $\begin{array}{l}\text { Tamaño } \\
\text { grupo* }\end{array}$ \\
\hline Directivo & Ministerio de Educación & \multirow[t]{4}{*}{ Nacional } & Individual & \\
\hline Funcionario & Icfes & & Individual & \\
\hline Directivo & Gremio educadores & & Individual & \\
\hline Miembro & Gremio educadores & & Individual & \\
\hline Directivo & Secretaría de Educación & \multirow[t]{18}{*}{ Departamental } & Individual & \\
\hline Funcionario & Secretaría de Educación & & Individual & \\
\hline Funcionario & Secretaría de Educación & & Individual & \\
\hline Funcionario & Secretaría de Educación & & Individual & \\
\hline Funcionario & Secretaría de Educación & & Individual & \\
\hline Supervisor & Secretaría de Educación & & Individual & \\
\hline Supervisores & Secretaría de Educación & & Grupal & 7 \\
\hline Directivo/académico & Universidad privada & & Individual & \\
\hline Directivo/académico & Universidad pública & & Grupal & 2 \\
\hline Académico & Universidad privada & & Grupal & 2 \\
\hline Académico & Universidad privada & & Individual & \\
\hline Académico & Universidad privada & & Individual & \\
\hline Académico & Universidad privada & & Individual & \\
\hline Académico & Universidad pública & & Individual & \\
\hline Asesor & Gremio educadores & & Individual & \\
\hline Directivo & Gremio educadores & & Individual & \\
\hline Directivo & Gremio educadores & & Individual & \\
\hline Directivo & Gremio educadores & & Individual & \\
\hline Director de núcleo & Secretaría de Educación & \multirow[t]{10}{*}{ Municipal } & Individual & \\
\hline Director de núcleo & Secretaría de Educación & & Individual & \\
\hline Funcionario & Gobierno local & & Individual & \\
\hline Rector & Institución educativa pública & & Individual & \\
\hline Rector & Institución educativa pública & & Individual & \\
\hline Rector & Institución educativa pública & & Individual & \\
\hline Docentes & Institución educativa pública & & Grupal & 31 \\
\hline Docentes & Institución educativa pública & & Grupal & 22 \\
\hline Docentes & Institución educativa pública & & Grupal & 36 \\
\hline Docente & Institución educativa pública & & Individual & \\
\hline
\end{tabular}

con categorías emergentes que surgieron de manera inductiva sobre los temas más relevantes mencionados por los actores entrevistados 
Nota: Las entrevistas individuales y grupales se llevaron a cabo dentro de una etnografía en la región y, por tanto, los criterios de elección de entrevistados incluyeron 1) disponibilidad, 2) recomendaciones de otros entrevistados y 3 ) apertura de espacios institucionales ( $p$. ej. talleres con docentes). No se incluyen los nombres y cargos específicos de ningún participante, en cuanto todos firmaron un consentimiento informado donde se les prometió confidencialidad en el uso de su nombre. Se buscó, en todo caso, dialogar con expertos en cada área, que incluyeron altos directivos relacionados con el debate de calidad educativa. Las entrevistas fueron en su mayoría semiestructuradas y los talleres con docentes siguieron metodologías participativas (un juego de votaciones a partir de los resultados de una encuesta sobre calidad educativa en dos municipios del departamento).

*Solo aplica a casos donde las entrevistas incluyeran a una o más personas.

Fuente: Elaboración propia a partir de registros de visitas y actividades en el departamento del Atlántico e instituciones nacionales entre agosto de 2014 y julio de 2015.

La presentación de resultados se hace a partir de una agrupación de tres grandes temas que ejemplifican dinámicas rectoras del proceso de descentralización, al menos según se puede capturar a partir del contraste entre testimonios de distintos actores educativos y que operan en diferentes niveles del sistema nacional de educación. Son los siguientes: 1) el fortalecimiento de capacidades locales, 2) la autonomía de instituciones frente al diseño de planes de estudio y 3 ) la rendición de cuentas del sistema educativo local de calidad. Siendo esta una lectura (básicamente) descriptiva, se busca evitar juicios de valor indicativos del alcance o el impacto de diferentes reformas. El uso de citas y el parafraseo de posturas expresadas por los entrevistados busca, por el contrario, resaltar acuerdos y conflictos que delimitan y potencian diferentes iniciativas de reforma educativa.

\section{Fortalecimiento de las capacidades locales}

Un primer subtema que sirve para ilustrar retos y visiones del proceso de descentralización educativa en Colombia es el del fortalecimiento de la Secretaría de Educación del Atlántico en su capacidad de actuar como interlocutor entre objetivos de política nacional y las necesidades concretas del territorio. Visto desde el ideal del proceso de descentralización (y de acuerdo con las funciones estipuladas por la legislación), esta es una instancia que debe ser capaz, no solo de coordinar y administrar, sino también de proponer rutas de política que se adapten a las particularidades de su contexto social, económico y cultural. En un diálogo con un grupo de supervisores de educación, ${ }^{10}$ podrían distinguirse dos etapas de este proceso a partir de la expedición de la Ley 715/2001, de 21 de diciembre. La primera (aproximadamente entre 2001 y 2006-2007) la catalogarían

\footnotetext{
${ }_{10}$ De acuerdo con el Decreto 907/1996, de 23 de mayo, los supervisores son funcionarios de la Secretaría de Educación, cuyo objetivo es hacer acompañamiento a directivos docentes y directores de núcleo frente al despliegue y la ejecución de programas y políticas educativas. Algunos de estos funcionarios llevan visitando semanalmente las instituciones educativas de la región por casi veinte años, lo cual los convierte en una fuente valiosa sobre la experiencia de las reformas educativas en el territorio.
} 
como el periodo de modernización ${ }^{11}$ y la segunda (a partir de 2007) como la era de un mayor enfoque en lo que sucede en las instituciones educativas.

A partir de las directivas nacionales que anunciaban la racionalización del gasto, la Secretaría de Educación del Atlántico inicia un proceso de restructuración, que incluye la sistematización de información administrativa (p. ej. sobre las plantas docentes) y la organización de sistemas de indicadores para monitorear resultados en torno a la calidad del sistema escolar. Ello implicaría también un esfuerzo en instituciones escolares a fin de alinear sus proyectos educativos institucionales y materializar los principios de autonomía escolar de la Ley General de Educación de 1994. Este proceso tomaría por sorpresa a rectores y directivos escolares, quienes hasta la fecha ejercían sus prácticas administrativas en medio de esquemas más informales de trabajo:

[Frente] a la reorganización de las plantas de personal y la consolidación de la autonomía curricular de los colegios para sus propios PEI [proyectos educativos institucionales], hay un hallazgo que es bueno tenerlo en cuenta; eso se generó en un contexto donde los profesores y los rectores en ese momento no estaban lo suficientemente capacitados y actualizados en cuestiones de gestión y refuerzos pedagógicos, entonces, las instituciones decayeron en muchas improvisaciones de planes de estudio de trabajo en el aula. (Supervisor de la Secretaría de Educación del Atlántico, comunicación personal, 20 de noviembre de 2014)

Para académicos de la región, la percepción de estas dificultades en el colegio se expresa aún hoy en instancias gubernamentales departamentales, en las cuales difícilmente se puede hablar de un sistema de información consolidado y, por tanto, preparado para elaborar tareas de monitoreo que respondan a parámetros ideales basados en una focalización adecuada del uso de capital humano y financiero desde la Secretaría de Educación del Atlántico. Así, por ejemplo, según declara uno de los expertos entrevistados,

[en] otro estudio que nosotros hacíamos, encontrábamos que eso que reportan las instituciones educativas directamente a la Secretaría y luego al sistema de educación nacional tiene una cantidad de errores interesantes [...] que termina impactando finalmente los recursos que tiene la institución educativa [...]. Cuando el Ministerio de Educación Nacional revisa las bases de datos y empieza a encontrar estudiantes fantasma [...] termina haciendo un descuento al giro que se descarga a la Secretaría de Educación, entonces ahí [se] nos cuestionaría el tema de ese fortalecimiento, porque lo que ha habido finalmente es que cada vez [...] los secretarios de educación tienen que [...] ser más creativos en cuanto a cómo administran los

${ }^{11}$ Por modernización los supervisores hacen referencia al proceso de fortalecimiento de capacidades tecnológicas y gerenciales de la secretaría de educación departamental para llevar a cabo tareas administrativas y de planeación de política pública. 
recursos partiendo de que ya tienen una deuda que viene siempre de los recortes de gestión. (Académico del departamento Atlántico, comunicación personal, 19 de septiembre de 2014)

Según lo manifiestan actores de diferentes niveles de gobierno, lo anterior puede ser reflejo de la persistencia en la confusión sobre responsabilidades y márgenes de acción con los que cuentan tanto el MinEducación como la Secretaría de Educación del Atlántico en el momento de determinar el uso de recursos para la educación. Desde esta segunda, los funcionarios que llevan décadas visitando colegios manifiestan que la falta de continuidad en iniciativas del Gobierno central generan dificultades en la implementación de medidas administrativas de política y así dan a entender una expectativa tácita de la forma en que el Gobierno territorial está supeditado a las iniciativas en la nación:

Las políticas son muy bonitas, se ven muy limpias cuando salen del nivel nacional, pero, cuando empiezan a bajar al nivel regional departamental y local, se empiezan a escurecer y a debilitar; uno dice, claro, bonita la presentación que hace la ministra, pero, cuando usted empieza a ver aquí, es otro cuento, y hay algo muy curioso que uno ve en las políticas y es que se trastocan cada vez que hay cambio de presidente de la república, cada vez que hay cambio de gobernador, cada vez que hay cambio de alcalde. (Supervisor de la Secretaría de Educación del Atlántico, comunicación personal, 3 de diciembre de 2014)

Desde el MinEducación, sin embargo, el problema es visto como un tema de falta de iniciativa desde el nivel territorial para el uso de recursos y la ejecución de iniciativas, dado que, a pesar de la existencia de lineamientos nacionales, los departamentos cuentan con autonomía para tomar decisiones que se ajusten a sus necesidades particulares:

Creo que no hay nada que vaya más en contra de la descentralización que pensar que la falta de una acción de unas secretarías es por algo que hace o no hace el ente central y creo que ese pensamiento demuestra el problema de la descentralización. Y es si la secretaría de educación $x$ no lo está haciendo bien es porque el Ministerio de Educación Nacional no le está ayudando a hacerlo bien. Eso me parece nefasto para el sistema, y creo que es lo que ha pasado, nosotros que hablamos con secretarios y líderes de calidad, particularmente yo, día a día uno siente eso, que es como "díganme qué hacer”, como no, al revés, “dígame usted qué quiere hacer”. (Directivo del Ministerio de Educación Nacional, comunicación personal, 10 de octubre de 2015)

La visión del gremio de educadores colinda, en parte, con la de la Secretaría de Educación del Atlántico, en cuanto expresan descontento frente a la forma que el MinEducación despliega estándares, a juicio de ellos, que poco dialogan con el territorio. 
En algo en que parecen discrepar - y en lo que, de cierta manera, coindicen más con la visión del Gobierno central- es que les gustaría tener un papel más activo en la generación de política educativa, según su visión de lo que se necesita en cada institución. Su queja es que los gobiernos no los tienen en cuenta, a pesar de su extensa experiencia en labores pedagógicas en campo:

Le estamos diciendo a la ministra que ella está hablando con todo el mundo menos con nosotros; ella no nos escucha a nosotros [...] a nosotros que nos desplazamos a todos los municipios y los departamentos nos toca venir acá o ir allá o de pronto nos llaman y no dicen lo que está pasando [...]. El Gobierno quiere un programa estandarizado igual para todo el mundo, que el del Chocó aprenda lo mismo que el de acá y el de acá aprenda lo mismo que el de Amazonas, donde tenemos costumbres, ideologías, economías, totalmente diferentes. Por eso, la alternativa, la propuesta de nosotros del PEPA. ${ }^{12}$ (Directivo del gremio de docentes del departamento del Atlántico, comunicación personal, 23 de octubre de 2014)

En suma, pese a las diferentes explicaciones ofrecidas por los actores representados, todos ellos coinciden en un diagnóstico sobre una modernización a medias de la Secretaría de Educación del Atlántico respecto de la generación de capacidades administrativas y de gestión, la cual enfrenta aún retos importantes para prestar el servicio educativo y diseñar políticas públicas. Siendo este un proceso que inició en la primera década del siglo XXI, se genera con ello evidencia, al menos indicativa, de un fallo relativo en la premisa de los modelos de elección pública, según la cual los mecanismos de traslado de poderes administrativos y de decisión a instancias territoriales debería responder más efectivamente a atender necesidades locales.

\section{La autonomía del territorio: el caso de las discusiones sobre la calidad de la educación}

El debate sobre el monitoreo y la evaluación de calidad educativa ha desatado una serie de controversias que pone de manifiesto la existencia de un discurso fragmentado entre diferentes niveles de gobierno sobre el uso de herramientas como las pruebas de Estado (en particular, las pruebas Saber 11). ${ }^{13}$ En este aspecto, la legislación estipula un margen de autonomía de los colegios (mediante la Ley 115/1994, de 8 de febrero) para

\footnotetext{
12 La sigla PEPA corresponde a Proyecto Pedagógico Alternativo, que, según la Federación Colombiana de Trabajadores de la Educación, plasma una visión más participativa en torno a la generación de políticas educativas desde el territorio.

${ }^{13}$ Esta aseveración no implica que el investigador asuma que calidad es igual a resultados en las pruebas de Estado. De momento, se analiza la posición institucional respecto de este tema.
} 
delinear planes de estudio (dentro de ciertos parámetros normativos ${ }^{14}$ ) adaptados a necesidades y características regionales. En este confluye la discusión sobre la naturaleza misma de la medición de posibles estándares de calidad (p. ej. desempeño escolar vs. niveles de satisfacción de la educación entre comunidades), y aunado a esto, la forma en que estos influyen (o no) en los contenidos curriculares de las instituciones educativas.

Para el Instituto Colombiano para la Evaluación de la Educación (Icfes), el tema es claro. Por un lado,

nosotros en el Icfes no creemos que el indicador de la calidad de la educación sea el examen; [este] es uno de tantos indicadores que existe... definir calidad en la educación es un tema muy complejo, porque depende de dónde usted se pare; es decir, usted se va para un colegio con unos estudiantes y unas exigencias económicas muy altas y ahí puede tener una definición de lo que es calidad de la educación para esa institución; pero, si usted se va para un colegio donde los muchachitos apenas llegan con un agua de panela, puede ser una cosa muy diferente [lo] que espera de la educación. (Funcionario del Icfes, comunicación personal, 20 de agosto de 2014)

\section{A su vez, el funcionario entrevistado agrega que}

el indicador que la gente ha utilizado son las notas y eso digamos que nosotros en el Icfes nunca hemos dicho que ese sea el indicador de calidad de la educación" (Funcionario del Icfes, comunicación personal, 20 de agosto de 2014).

De hecho, en el plano conceptual, esta institución ha sido enfática en que su objetivo al publicar resultados agregados de exámenes por colegio es generar un parámetro para que cada institución se vea a sí misma, se compare con instituciones pares y genere incentivos para la toma de decisiones (en medio de su autonomía), a fin de revisar sus prácticas pedagógicas. Y en parte, así lo reconoce la Secretaría de Educación del Atlántico:

Han sido claros, por lo menos el Icfes, en decir que [esta] es una evaluación de algunos apartes del proceso de educación [...] por ejemplo de las competencias matemáticas, unas competencias de lenguaje generales mas no de [...] todo el proceso [...] educativo [pues este] implica la parte convivencial [sobre el] cómo se relaciona el niño con el otro [y] cómo responde a unas necesidades sociales familiares culturales. (Funcionario de la Secretaría de Educación del Atlántico, comunicación personal, 23 de septiembre de 2014)

\footnotetext{
${ }^{14}$ Según el artículo 23 de la Ley 115/1994, de 8 de febrero, las instituciones cuentan con plena autonomía para definir el $20 \%$ de su plan de estudios (por fuera de los grupos de áreas obligatorias). Sin embargo, existe libertad en la institución educativa (art. 77) en cuanto a la aplicación de métodos de enseñanza y organización de actividades formativas.
} 
Sin embargo, como reconoce una funcionaria, este no es el mensaje que se recibe en los colegios. A hora de la verdad, sostiene,

cuando vamos a hablar de la calidad de los establecimientos educativos [...] [y el] Ministerio [de Educación] va a medir la calidad de la educación, la medimos mucho desde las pruebas Saber" (Funcionaria de la Secretaría de Educación del Atlántico, 7 de noviembre de 2014).

Dialogando con un alto directivo en el MinEducación, esta narrativa parece coincidir con la visión que se les da a las pruebas de desempeño como un indicador de resultado y de medición de calidad. Desde tal perspectiva, este debería ser, además, una medición útil para tomar decisiones sobre asignación de recursos y focalización de programas: ${ }^{15}$

Probablemente, los académicos no [lo] ven, [pero] hay un sentido muy perverso en que el Sistema General de Participaciones se distribuya a partir de matrículas, por ejemplo, no a través de calidad, o sea, esto es un sistema que históricamente se ha pensado en términos de inputs, y tú vas a la región, y es “que no me dan”, “que me falta”, o sea, cómo, y nunca se piensa en términos de resultados [...] nunca se habla de y “yo ċcuánto le puedo exigir?”. Históricamente, eso sería superbonito que lo vieras, cómo ha incrementado la inversión en educación, y cómo, a pesar de eso, la calidad sigue estancada. (Directivo del MinEducación, comunicación personal, 10 de octubre de 2015)

Los gremios educativos, por su parte, han sido insistentes en mostrar su descontento frente a nociones de productividad inmersas en las pruebas, y que celebran otros actores. Un ejemplo de ello se ve reflejado en la manera en que la Federación Colombiana de Trabajadores de la Educación (Fecode) expresa una preocupación en torno a la regulación de la educación "según las necesidades del mercado" (miembro de Fecode, comunicación personal, 11 de septiembre de 2014) o cómo, desde la agremiación de educadores del departamento del Atlántico, se afirma que este no es más que un mecanismo que es utilizado por el MinEducación para poner límites al ascenso en el escalafón docente:

INVESTIGADOR: ¿Cuál es el objetivo del Icfes en Colombia con la prueba Saber 11? ENTREVISTADO: Sencillo. Para determinar qué maestros sirven y qué maestros no. Simplemente, lo están poniendo como una medida [para definir a] qué maestros se les puede subir el sueldo y a qué maestros no se les puede subir el sueldo. (Miembro del gremio de docentes del departamento del Atlántico, comunicación personal, $1 .^{\circ}$ de septiembre de 2014)

\footnotetext{
15 Práctica que se da, por ejemplo, en la definición de territorios para la implementación de políticas como el Programa Todos a Aprender, iniciativa en la cual los docentes ejemplares visitan colegios para retroalimentar la práctica de sus pares.
} 
En medio de esta controversia, sin embargo, y tal como se reconoce de la Secretaría de Educación del Atlántico, parece llegar un mensaje confuso a las instituciones educativas, las cuales, pese a gozar de autonomía para definir parte de sus contenidos y esquemas pedagógicos, terminan adaptando sus planes de estudio a fin de preparar (casi exclusivamente) a los estudiantes para las pruebas de Estado. Es una práctica común, al menos en las instituciones visitadas, que una porción importante del tiempo de clase en los últimos años sea destinada a hacer simulacros con el propósito de que los estudiantes aprendan técnicas para resolver preguntas estandarizas. Al respecto, Grindle (2004) reporta que esta es una práctica ampliamente normalizada en América Latina. De hecho, como lo permite entrever una anécdota narrada por uno de los rectores de los colegios, este se convierte en un tema central en la pugna por la obtención y la ejecución de los recursos de calidad:

Como no se puede hacer con fondos de servicios educativos el pago para pruebas Icfes, la Alcaldía da eso, pero la Alcaldía da vueltas, y [empezó] en abril o mayo, ya se había perdido medio año, y lo segundo es que la institución debe garantizar al muchacho es que desde primaria hasta el grado once debe tener un tipo de evaluación coherente con lo que hace el Icfes. (Rector de colegio, comunicación personal, 12 de noviembre de 2014)

La conclusión parcial que se desprende de estas visiones es que la autonomía de facto de las instituciones escolares (incluso en los márgenes establecidos por la normativa) parece estar amenazada por la existencia de unos estándares que tienen una mayor injerencia de la prevista sobre los contenidos escolares territoriales. Según denuncian algunos actores, existe una contradicción tácita entre lo que estipula el nivel central del sistema de evaluación y la forma en que los indicadores de calidad terminan fijando los parámetros a los cuales todos los actores deben alinearse. Esto, visto desde la óptica crítica a elección pública, termina contradiciendo preceptos de una prestación de un servicio que se acerque más a las necesidades concretas (p. ej. según contextos, visiones culturales, etc.) de sus usuarios.

\section{¿Qué pasa con la rendición de cuentas en la ejecución de los recursos de calidad?}

Un tercer tema que resulta ilustrativo de los retos pendientes de la descentralización educativa, al menos según las visiones expresadas por diferentes actores, es el de la rendición de cuentas en torno a la ejecución de recursos territoriales. De acuerdo con las predicciones teóricas del esquema descentralizador, este problema debería irse superando en la medida en que exista una mayor cercanía entre gobernantes y gobernados. Al tiempo, como se pudo ver en algunas de las recomendaciones de expertos, resulta deseable permitir a los territorios contar con recursos libres de burocracia regional para que resuelven más efectivamente sus problemas locales. 
Uno de los aspectos visibles que surgen del diálogo con expertos es que, como es apenas previsible, la descentralización del sistema educativo no puede estar aislada del funcionamiento del Estado (esta es una de las principales tesis del trabajado de López y Jiménez, 2016). Según lo contempla la normativa, parte del Sistema General de Participaciones que se destina a gratuidad es girado directamente a alcaldes, quienes a su vez deben conformar comités de calidad (compuestos por miembros de su Gobierno local y representantes de la comunidad educativa de cada municipio) para definir la forma en que se deben utilizar estos recursos. Un primer reto será, por ende, verificar que, en efecto, se conformen estas instancias, y que funcionen de acuerdo con parámetros avalados por mecanismos de control. Sin embargo, según narra un funcionario de la Secretaría de Educación del Atlántico,

\begin{abstract}
si un comité de calidad no está funcionando, nosotros debemos notificar a las ías [es decir, a las contralorías y procuradurías] para que hagan ese seguimiento. Nosotros lo requerimos al alcalde, pero quien sanciona o quien llama la atención son [los mecanismos de control] [...] pero nosotros no hemos entrado en ese proceso [...] porque se demora más el proceso [que] mirar la calidad. (Funcionario de la Secretaría de Educación del Atlántico, comunicación personal)
\end{abstract}

De hecho, los rectores, en general, manifiestan que resulta difícil hacer rendir cuentas a los alcaldes sobre el uso de estos recursos. Esta situación es constatada por opiniones expresadas en los municipios del sur del departamento, según las cuales limita la materialización de proyectos educativos que involucren a comunidades e instituciones educativas:

\footnotetext{
Hay un desconocimiento total de cuáles son las inversiones hechas y proyectadas [...] En este momento, ahí sí siento que, como institución educativa, remamos contra la corriente, porque nosotros vamos apuntando hacia una dirección y sentimos que la Administración municipal, que debe ser nuestro aliado, va en otra. Entonces, eso ha sido también una de las grandes o la mayor dificultad. [...] Tenemos [por ejemplo una] dificultad con el SENA [Servicio Nacional de Aprendizaje] [porque] no tenemos espacio y una de las propuestas que se le hizo al alcalde en su debido momento era acondicionar una sede que está aquí abandonada y entregarme un lote. (Rector de colegio del sur del Atlántico, comunicación personal, 28 de octubre de 2014)
}

En otros casos similares, los rectores afirman que la misma falta de control sobre el uso de los recursos tiene como consecuencia la prestación de servicios que resultan poco pertinentes para cumplir metas institucionales. Así, por ejemplo,

él [alcalde] hace lo que le da la gana con esa plata, principalmente, las capacitaciones que mandan al mismo [contratista] que coge los estudiantes de 10 o de 11 [y] les da una charla. 
Eso es todo el aporte que le hace a las escuelas y ya el discurso de los estudiantes se lo saben de memoria y ya no quieren escuchar más. (Rector de colegio del sur del Atlántico, comunicación personal, 28 de octubre de 2014)

Repasando el estudio comparado de Grindle (2004) sobre la experiencia de las reformas educativas en el continente, este deja una enseñanza clara: la operación de mecanismos de mercado - al menos en el contexto latinoamericano- requiere más, y no menos, Estado. Muchos de los trabajos econométricos que operan bajo el principio de aislar el efecto de variables trivializan este hecho. Las expresiones aquí expresadas manifiestan que, por el contrario, sin un funcionamiento efectivo de otros poderes, y otros sectores del Estado, se hace difícil concertar iniciativas educativas desde el territorio.

\section{Comentarios finales}

Montoya-Vargas (2014) señala, en su análisis sobre el estado de los estudios de currículo en Colombia, que siempre que el MinEducación quiere mejorar la calidad educativa recurre a fórmulas similares. Lo que sugiere su trabajo es que estas iniciativas se reproducen en el tiempo sin mostrar los avances esperados. Tal tipo de diagnósticos dialoga con las visiones expresadas en este artículo, en la medida en que, al menos en el departamento del Atlántico, las iniciativas de política educativa, desde la perspectiva de distintos actores sociales, y desde diferentes niveles de gobierno, se ven frustradas por una descentralización política y administrativa (en el mejor de los casos) limitada.

Este artículo contribuye a la literatura sobre la descentralización en Colombia, vista desde la óptica de la reforma educativa. En particular, los elementos conceptuales, ontológicos y analíticos expuestos permiten dar un paso crítico en al menos dos direcciones necesarias: una mirada al proceso descentralizador desde las complejidades del territorio y una alternativa metodológica en medio de una literatura que parece haber agotado su capacidad de decir algo más sobre el porqué (y hasta qué punto) se han cumplido las promesas de la Constitución Política de 1991. La experiencia del departamento del Atlántico genera evidencia de cómo los mecanismos comúnmente defendidos por expertos y especialistas influenciados por los marcos de referencia de la elección pública -como el mejoramiento de las capacidades y los incentivos de gobiernos locales para atender necesidades del territorio- distan de operar de la manera esperada.

Ahora bien, algunos lectores podrían reaccionar de manera crítica a estos hallazgos al señalar que las dinámicas identificadas son de pleno conocimiento en el debate público y entre estudiosos del tema. Muchos increparían, por tanto, la utilidad o la novedad en la aplicación del realismo crítico en estudios de este tipo. Al respecto, resulta relevante citar las palabras de los autores de un reciente trabajo que tuvo una 
importante acogida entre expertos del gremio educativo: "A pesar de su importancia, [...] la propuesta de política que hace nuestro estudio no radica en la dimensión socioeconómica (de los hogares) [...] [en cuanto] el espectro para modificar las variables de contexto [...] es limitado desde la perspectiva de política educativa" (las cursivas mías") (García, Maldonado, Perry, Rodríguez y Saavedra, 2014, p. 91). El punto por resaltar es cómo las decisiones arbitrarias de algunos investigadores inspiradas por el sesgo cuantitativo (solo se estudia lo que se puede medir, solo se recomienda lo que se considera posible hacer) generan una brecha exagerada entre las construcciones teóricas y las realidades sociales en estudio. El realismo crítico aporta a disminuir esta brecha al otorgar peso y valor epistemológico a visores metodológicos cualitativos que permiten capturar la complejidad como parte de un ejercicio académico científico y riguroso. El llamado de atención para la comunidad académica es a establecer un límite entre la investigación científica y la visión del hacedor de política (sobre lo que se supone que es posible hacer), sin la cual es difícil estudiar las causas reales de los retos contemporáneos de política pública.

Frente a este estudio, es importante insistir en que las conclusiones esbozadas en el artículo distan de contar con un poder causal. La postura realista que inspira el análisis así lo anticipa; las opiniones expresadas por actores políticos, quienes viven en entornos que pueden modificar, pero cuyas propiedades estructurales existen (e influencian su comportamiento) a pesar de que estos no sean completamente conscientes de ello, implica una visión de la causalidad social que requiere un estudio diferenciado entre la complejidad de las interacciones sociales observadas en un momento temporal y geográfico específico y las estructuras materiales y culturales que preexisten y condicionan (mas no determinan) la acción (o la causa eficaz) colectiva de individuos y grupos sociales (Archer, 2009). La mediación entre estructura y agencia es un proceso analítico que requiere ambas visiones y un ejercicio de teorización que, sin embargo, se escapa del espectro de este artículo.

Y entonces, ¿̇qué explica la descentralización (al menos) limitada que perciben los actores entrevistados? Esa es la pregunta a la que (retomando el párrafo anterior) busca responder la agenda de investigación más general en la cual se enmarca este trabajo. De momento, es posible pensar en algunas hipótesis inspiradas en estudios sociológicos comparados como el de Parada (2013) y el Soifer (2015), quienes sugieren la remanencia de un lejanía histórica entre las dinámicas políticas, sociales y culturales del centro (desde donde se despliega la descentralización) y la Costa Caribe. Ello se ve reflejado en la historia de un país forjado a través de pugnas entre élites locales y nacionales, donde el interés construido en torno a la educación ha estado marcado por la existencia de pocos incentivos para consolidar un proyecto de Estado nación. La pregunta, no obstante, es empírica y será abordada en las siguientes fases analíticas de este proyecto. 


\section{Referencias}

Archer, M. S. (2009). Teoría social realista: el enfoque morfogenético. Santiago de Chile: Universidad Alberto Hurtado.

Archer, M. S. (2013). Social origins of educational systems. Londres: Routledge.

Barrera Osorio, F. (2005). La política de educación en Colombia 1957-2004. Recuperado de http://www.repository.fedesarrollo.org.co/handle/11445/896

Barrera Osorio, F., Maldonado Carrizosa, D. y Rodríguez Orgales, C. (2014). Calidad de la educación básica y media en Colombia: diagnóstico y propuestas. En A. Montenegro y M. Meléndez (comps.), Equidad y movilidad social: diagnósticos y propuestas para la transformación de la sociedad colombiana (pp. 239-330). Bogotá: Universidad de los Andes.

Chamorro Miranda, D. J., Borjas, M. P. y Valencia Cobo, J. (2013). Básica y media. En Situación de la educación en el Caribe colombiano 2008-2011 (pp. 53-103). Barranquilla: Universidad del Norte/Observatorio de Educación del Caribe Colombiano.

Decreto 907/1996, 23 de mayo 23, por el cual se reglamenta el ejercicio de la suprema inspección y vigilancia del servicio público educativo y se dictan otras disposiciones.

Easton, G. (2010). Critical realism in case study research. Industrial Marketing Management, 39(1), 118-128.

Edwards Jr, D. B. y Brehm, W. C. (2015). The emergence of Cambodian civil society within global educational governance: A morphogenetic approach to agency and structure. Journal of Education Policy, 3o(2), 275-293.

Emmel, N. (2013). Sampling and choosing cases in qualitative research: A realist approach. Londres: Sage.

Faguet, J.-P. (2014). Decentralization and Governance. World Development, 53, 2-13.

Faguet, J.-P. y Sánchez, F. (2008). Decentralization's effects on educational outcomes in Bolivia and Colombia. World Development, 36(7), 1294-1316.

Faguet, J.-P. y Sánchez, F. (2014). Decentralization and access to social services in Colombia. Pubic Choice, 16o(1), 227-249.

Falleti, T. (2005). A sequential theory of decentralization: Latin American cases in comparative perspective. American Political Science Review, 99(3), 327-346.

García, S., Maldonado, D., Perry, G., Rodríguez, C. y Saavedra, J. E. (2014). Resumen y precisiones sobre Tras la excelencia docente: cómo mejorar la calidad de la educación para todos los colombianos. Revista Colombiana de Educación, 67, 89-105. 
García Villegas, M., Espinosa Restrepo, J. R., Jiménez Ángel, F. y Parra Heredia, J. D. (2013). Separados y desiguales: educación y clases sociales en Colombia. Bogotá: Dejusticia.

Gobernación del Atlántico (2014). Plan de Desarrollo 2012-2015 José Antonio Segebre Berardinelli. Barranquilla: Gobernación del Atlántico.

González Delgado, M. (2013). Teoría social realista y la construcción del sistema educativo estatal en España: algunas propuestas para el análisis del cambio educativo. Revista Mexicana de Investigación Educativa, 18(59), 1213-1239.

Grindle, M. S. (2004). Despite the odds: The contentious politics of education reform. Princeton, New Jersey: Princeton University Press.

Grindle, M. S. (2007). Going local: decentralization, democratization, and the promise of good governance. Princeton, New Jersey: Princeton University Press.

Henrich, J., Boyd, R., Bowles, S., Camerer, C., Fehr, E., Gintis, H. y McElreath, R. (2001). Search of homo economicus: Behavioral experiments in 15 small-scale societies. The American Economic Review, 91(2), 73-78.

Henríquez Algarín, H. de J. (2013). Politicas públicas en educación y desarrollo en la Región Caribe. Revista de Economía del Caribe, 12, 217-246.

Hoyos, D. y Ceballos, M. (2004). Tendencias del comportamiento electoral y descentralización en los municipios de Colombia, 1988-20oo. Londres: Crisis States Programme.

Iregui, A. M., Melo, L. y Ramos, J. (2006). Análisis de eficiencia de la educación en Colombia. Revista de Economía del Rosario, 10(1), 21-41.

Krauss, A. (2016). The scientific limits of understanding the (potential) relationship between complex social phenomena: The case of democracy and inequality. Journal of Economic Methodology, 23(1), 97-109.

Ley 115/1994, de 8 de febrero, por la cual se expide la Ley General de Educación.

Ley 715/2001, de 21 de diciembre, por la cual se dictan normas orgánicas en materia de recursos y competencias de conformidad con los artículos 151, 288, 356 y 357 (Acto Legislativo 01 de 2001) de la Constitución Política y se dictan otras disposiciones para organizar la prestación de los servicios de educación y salud, entre otros.

López, C. y Jiménez, F. (2016). De lo nacional a lo local: las reformas a la descentralización y las capacidades de estatalidad a nivel local. En C. López, iAdiós a las FARC! ¿Y ahora qué? Construir ciudadanía, Estado y mercado para unir las tres Colombias (pp. 477-513). Bogotá: Debate. 
Martinez-Vazques, J. (2011). The impact of fiscal decentralization: Issues in theory and challenges in practice. Manila: Asian Development Bank.

Ministerio de Educación Nacional (2009). Guía 33: Organización del sistema educativo: conceptos generales de la educación preescolar, básica y media. Bogotá: Ministerio de Educación Nacional.

Montoya-Vargas, J. (2014). The field of curriculum studies in Colombia. En W. F. Pinar (ed.), International handbook of curriculum research (vol. 2, pp. 134-150). Nueva York: Routledge.

Parada Corrales, J. (2013). Instituciones, desarrollo y regiones: el caso de Colombia. Barranquilla: Universidad del Norte.

Parra Heredia, J. D. (2016). Realismo crítico: una alternativa en el análisis social. Sociedad y Economía, 31, 215-238.

Parra Heredia, J. D. (2018). Critical realism and school effectiveness research in Colombia: The difference it should make. The British Journal of Sociology of Education, 39(1), 107-125.

Patomäki, H. (2009). How to tell better stories about the history and future of Global Political Economy. Review of International Political Economy, 16(2), 309-320.

Pawson, R. (1996). Theorizing the interview. The British Journal of Sociology, 47(2), 295-314.

Pino, J. F. (2017). Entre democracias y autoritarismos: una mirada crítica al estudio de la democracia subnacional en Colombia y Latinoamérica. Colombia Internacional, 91, 215-242.

Priestley, M. y Miller, K. (2012). Educational change in Scotland: Policy, context and biography. The Curriculum Journal, 23(1), 99-116.

Ramírez, M. T. y Téllez, J. P. (2007). La educación primaria y secundaria en Colombia en el siglo XX. En J. Robinson y M. Urrutia (eds.), Economía colombiana del siglo $X X$ : un análisis cuantitativo (pp. 459-517). Bogotá: Fondo de Cultura Económica.

Rees, C. y Gatenby, M. (2014). Critical realism and ethnography. En P. K. Edwards, J. O’Mahoney y S. Vincent (eds.), Studying organizations using critical realism: A practical guide (pp. 132-147). Oxford: Oxford University Press.

Said-Hung, E. (ed.) (2014). Estado de la educación en el Caribe colombiano 2011-2013. Barranquilla: Universidad del Norte/Observatorio de Educación.

Sánchez Torres, F. y Pachón, M. (2013). Descentralización, esfuerzo fiscal y progreso social en Colombia en el nivel local, 1994-2009: ¿’por qué importa la política nacional? Documento CEDE, 38. 
Soifer, H. D. (2015). State building in Latin America. Nueva York: Cambridge University Press.

Verger, A., Bonal, X. y Zancajo, A. (2016). What are the role and impact of public-private partnerships in education? A realist evaluation of the Chilean education quasimarket. Comparative Education Review, 6o(2), 223-248.

Villar, L. (dir.) (2016). Sistema General de Participaciones y su vínculo con las políticas e incentivos dirigidos a la excelencia docente y el mejoramiento continuo de la calidad de la educación. Bogotá: Fundación para la Educación Superior y el Desarrollo.

Vivas Pacheco, H. (2009). Educación, desigualdad y democracia. Sociedad y Economía, $16,173-188$.

Willmott, R. (1999). Structure, agency and the sociology of education: Rescuing analytical dualism. British Journal of Sociology of Education, 2O(1), 5-21. 
